\title{
STONE DENSITY DETECTED BY NON CONTRAST COMPUTED TOMOGRAPHY (LOW DOSE) IS A PREDICTOR OF SUCCESSFUL OUTCOME OF RENAL STONE CLEARANCE BY EXTRA CORPOREAL SHOCK WAVE LITHOTRIPSY
}

\author{
MOHAMMAD AL AMIN ${ }^{1}$, MD. SAIFUL ISLAM ${ }^{1}$, S.M. YUNUS ALI ${ }^{1}$, MD. GOLAM MOWLA CHOUDHURY $^{1}$, \\ MOHAMMED MIZANUR RAHMAN ${ }^{2}$
}

${ }^{1}$ Dept. of Urology, BSMMU, 2Dept. of Urology, NIKDU, Dhaka

\begin{abstract}
Objective- To evaluate the usefulness of measuring stone density in Hounsfield Unit by Low-dose Non Contrast Computed Tomography scan in predicting the outcome of extracorporeal shockwave lithotripsy for renal stone clearance.

Materials \& Methods - A total of 96 patients with renal stone size d" $20 \mathrm{~mm}$ attending at the OPD of BSMMU were included in this study. The outcome measures were complete clearance of stone, number of ESWL sessions and number of shock waves required to become stone free.

Result- The mean size of the stone was $1.8 \pm 0.3 \mathrm{~cm}$. The mean stone density was 663.7 $\pm 69.8 \mathrm{HU}$. $25 \%$ of the patients underwent 2 sessions of ESWL, $52.1 \%$ three sessions and $22.9 \%$ more than 3 sessions. Of the patients $83.3 \%$ were successfully cleared of their stone. The mean number of shock waves $6689.2 \pm 268.4$ required for stone fragmentation of d" $750 \mathrm{HU}$ and $9945 \pm 375.7$ required $>750 \mathrm{HU}$ stone density respectively. $85.5 \%$ of the patients with stone density d" $750 \mathrm{HU}$ needed d" 3 sessions to become stone-free; whereas only $55.5 \%$ of the patients with stone density $>750 \mathrm{HU}$ became stone-free in d" 3 sessions. $14.5 \%$ patients needed $>3$ sessions of ESWL with stone density of e" $750 \mathrm{HU} .78 .8 \%$ of the patients with stone density d" $750 \mathrm{HU}$ exhibited complete clearance of stone as opposed to $37.5 \%$ of those with stone density $>750 \mathrm{HU}$. The chance of having complete stone clearance is 6-fold (95\% Cl $=1.9-19.4)$ higher in patients with low density stone ( $d$ " $750 \mathrm{HU}$ ) than that in patients with high density stone (d" $750 \mathrm{HU})(p=0.002)$.
\end{abstract}

Conclusion-In conclusion a stone density less than $750 \mathrm{HU}$ should be treated with ESWL as first choice of treatment.

Key wards- Stone, Density, ESWL

Bangladesh J. Urol. 2016; 19(2): 90-97

\section{Introduction:}

The incidence of nephrolithiasis is reported to be increasing across the world[1,2]. This increase is seen regardless of factors such as age, sex and race. However, obesity, diminished intake of fluid, increased consumption of calcium, sodium, oxalate, animal protein are considered the most important risk factors for renal

Correspondences: Dr. Mohammad Al Amin, Dept. of Urology, BSMMU, Dhaka stone formation[3,4]. Because of its high frequency, urolithiasis is of particular concern of health economics as well as increased of total annual cost. Therefore, scheduling the management of the urolithiasis is of utmost importance in decreasing the subsequent cost after diagnosis.

Since 1980, extracorporeal shock wave lithotripsy is the first-line of treatment for renal stones of $<2 \mathrm{~cm}$ in diameter[5]. Analyzing different series, its success rates 
varies from $60-99 \%[6]$. However, the outcome of ESWL depends on many factors, like stone size, composition, fragility, the shock wave generator, the presence of obstruction or infection and the distance from the skin to stone[7.80]. After the introduction of the concept of fragility, stone composition has emerged as the main factor influencing the efficacy of ESWL[9].

Non Contrast CT (NCCT) has long been used to evaluate causes of radiolucent filling defects using measurements of substance density in Hounsfield units (HU) to distinguish calculi from tumours or blood clot[10,11]. As it provides greater density discrimination than a conventional plain skiagram of abdomen, it is now the preferred method to evaluate which patients with urinary calculi will have successful outcome following ESWL ${ }^{[12]}$. Its ability to detect density differences as low as $0.5 \%$ has been exploited to determine the composition and fragility of urinary stones ${ }^{[9,13]}$. The density of stone varies with composition and affects the fragility of a calculus, which ultimately governs the clinical outcome in ESWL. Therefore, knowing the fragility of a calculus before ESWL is of utmost importance to increase the efficacy and reduce the number of hospital visits and cost of treatment.

The stone density and ability of a stone to resist ESWL is based in the part on the composition as well as microcrystal of the stone. Stone composed of calcium oxalate dihydrate, magnesium ammonium phosphate, uric acid tend to be softer and to fragment more easily with ESWL. Stone composed of calcium oxalate monohydrate, cystine stone are less susceptible to ESWL. The more crystalline dehydrate stones are easiest to break. The monohydrates stones are much harder to break. Therefore, Low density renal stone easily fragments by ESWL and High density renal stone difficult to fragments or not fragmented by ESWL[13].

Repeated ESWL use causes various complications like peri-renal, subscapular or intra-renal hemorrhage, post ESWL hematuria, recurrent urinary tract infection, stone fragments inducing obstructive uropathy and hypertension.

For that reason to reduce the number and complication of the stone diseases, this study proposed to find out the outcome of treatment of renal stone clearance by extra corporeal shockwave lithotripsy after evaluating the stone density detected by non-contrast computed tomography (Low-dose).

\section{Materials and Method:}

This prospective observational type of study was carried out in the Department of Urology, BSMMU, Dhaka over a period of 18 months between January 2013 to June 2014 with patients of renal stone attending at the department.

Total 96 adult patients were selected by standard statistical sampling formula and inclusion \& exclusion criteria, with solitary renal stone (size $>5 \mathrm{~mm}$ and d" 20 $\mathrm{mm}$ ) except in lower calyx and residual single stone after surgery were included in this study in. All patients were included with informed written consent.

Data were collected using a structured questionnaire (Research Instrument) containing all the variables of interest. The questionnaire were finalized following pre testing. Collected data were checked daily and edited. Data were processed and analysed using computer software SPSS (Statistical Package for Social Sciences). The test statistics used for analysis of data are Chi-square Test (for comparison of categorical data between groups) and Student's t-Test (for comparison of continuous data between groups). The association between stone density and outcome ESWL were tested by means of univariate analyses. Level of significance was set at 0.05 and $p<0.05$ were considered significant.

\section{Detailed procedure:}

All patients were evaluated by: Hematological test like $\mathrm{CBC}$ and Coagulation profile, Biochemical tests like S. Creatinine, Blood urea, Urine RME and C/S, Plain X ray of KUB region, USG of whole abdomen, Intravenous urograpy, Non contrast computed tomography (NCCT).

Non Contrast CT scans procedure:

Pre procedural radiological evaluation included plain $\mathrm{x}$ ray of the kidney-ureter-bladder region and non contrast CT of KUB region on a multi-slice CT scanner. All images were obtained with a $4^{\text {th }}$ generation Hitachi CT scanner (appendix-v) without intravenous or oral administration of contrast medium. The section thickness and interval was 3 to $5 \mathrm{~mm}$. Images were obtained with 0.8 -second gantry rotation by Low-dose CT protocol that is $120 \mathrm{kVp}$ and $80 \mathrm{~mA}$. ( BSMMU CT protocol for abdomen is 140 $\mathrm{kVp}$ and $150 \mathrm{~mA}$ ). Hence $\mathrm{HU}$ for each stone were determined on the pretreatment NCCT and only single shot will be taken. Stone size, stone location (pelvis, calyx), stone attenuation values (Hounsfield units) and skin to stone distance were recorded. The lowest, highest and most common attenuation values were recorded and the mean calculus attenuation value was 
calculated. The skin-to stone distance (SSD) was calculated by measuring three distances from the stone to the skin at $0^{\circ}, 45^{\circ}$ and $90^{\circ}$ using radiographic calipers, and the average of these values was calculated to represent SSD for each stone. Whole procedure was done in the department of radiology and imaging of BSMMU and reported by expert radiologist in that department.

\section{ESWL Procedure:}

ESWL monotherapy with $3^{\text {rd }}$ generation Siemens Lithoskop lithotripter was used to treat all the enrolled patients with structured procedure followed. The number of shock waves to be delivered to the stone by expert operator during each session. Standard number of shock waves 2000 to 2500 with frequency $90 \mathrm{P} /$ min per session and energy setting of $3.5 \mathrm{KV}$ was applied in each session for lithotripsy. A change in stone size or outline or separation of stone fragments indicated fragmentation which was observed by fluoroscopy. The procedure was terminated if satisfactory fragmentation was noted before 3 session of ESWL or no change in stone size and outline up to 3 session of ESWL. All patients were hospitalized during ESWL procedure and served as day care service. All patients were under antibiotic prophylaxis during the procedure.

\section{Post ESWL evaluation:}

Patients were advised to come after 4 weeks with a plain X-ray of KUB region. In the follow up study, history, clinical examination and relevant investigations like urine routine examination, culture and sensitivity were done and data on post ESWL clearance were recorded in data sheet for assessing the outcome. ESWL success is defined as patients being stone free or with remaining stone fragments of $<4 \mathrm{~mm}$ after three session which is consider as clinically insignificant residual fragments (CIRF) consider as the success of ESWL and complete stone clearance. Remaining fragments of $>4 \mathrm{~mm}$ or nonfragmented stone were considered as ESWL failure as well as for other treatment option should be choice ${ }^{[14]}$.

\section{Results and observation:}

The result shows that mean age of the total 96 patients was $49.4 \pm 8.2$ years and the youngest and the oldest patients were 35 and 68 years old respectively (Table I). The majority ( $77.1 \%)$ of the patients were male (74) with male to female (22) ratio is 3:1. Out of 96 patients $36 \%$ patients having normal $\mathrm{BMI}$ and needed d" 3 sessions of ESWL where as $64 \%$ patients having overweight needed $>3$ sessions of ESWL. In relation to
$\mathrm{BMI}$, complete stone clearance between overweight and normal was $63.8 \%$ and $36.2 \%$. But no stone clearance between these was $60.0 \%$ and $40.0 \%$. p-value was significant. The association between skin to stone distance and number of ESWL session, shows that the mean skin to stone distance in case of d"3 ESWL session was $84.2 \pm 2.9$ and $>3$ ESWL was $87.9 \pm 1.8$ respectively (Table-II). Statistically significant difference present between these groups ( $p$-value $<0.05$ ). The stone characteristics associated with stone clearance, shows $55.2 \%$ of the patients had left kidney and $44.8 \%$ had right kidney stone involvement of which $62.5 \%$ of the patients had stone in the renal pelvis and $37.5 \%$ of the patients had calyceal stone. The mean size of the stone was $1.8 \pm 0.3 \mathrm{~cm}$ (range: $0.98-2 \mathrm{~cm}$ ). The mean stone density was $663.7 \pm 69.8 \mathrm{HU}$ (range: $133-1485$ $\mathrm{HU}$ ). The location of stones in calices, this study showed no significant difference was observedin complete stone clearence in relation to site and location of stones $(p>$ $0.05)$. The mean of stone density in complete stone clearence was $478.3 \pm 38.2$ (range: $133-750 \mathrm{HU}$ ) and incomplete stone clearance was $943.6 \pm 50.9$ (range: $751-1485 \mathrm{HU})$ that was statistically significant $(p<$ 0.001).

The number of ESWL session and stone size in this study shows that $25 \%$ of the patients underwent 2 sessions where stone size was $0.98 \mathrm{~cm}$ to $1.10 \mathrm{~cm}$. $52.1 \%$ of patients needed three sessions where stone size $1.11 \mathrm{~cm}$ to $1.94 \mathrm{~cm}$ and $22.9 \%$ of patients needed more than 3 sessions where stone size were $1.95 \mathrm{~cm}$ to $2.0 \mathrm{~cm}$. $83.3 \%$ of the patients successfully cleared off stone by ESWL.

The relation between the number of ESWL session and stone density shows that $85.5 \%$ of the patients with stone density d" $750 \mathrm{HU}$ needed 3 or less session to have their stone cleared and the rest (14.5\%) needed > 3 session with same density. In patients with stone density $>750 \mathrm{HU}, 55.5 \%$ needed 3 or less session and $44.5 \%$ needed $>3$ sessions. $p$-value is significant ( $p<0.001)$ (Table-III).

The association of the amount of shock wave and stone density shows that $79.7 \%$ patients having stone density d"750 needed d" 7500 shock wave of ESWL and $20.3 \%$ patients needed $>7500$ shock wave. $74.1 \%$ of patients having stone density $>750$ needed d" 7500 shock wave and $25.9 \%$ of patients needed $>7500$ shock wave of ESWL. The mean shock wave is $6689.2 \pm 268.4$ in d"750 HU stone and $9945.2 \pm 375.7$ in $>750 \mathrm{HU}$ stone. There was significant difference in amount of shock wave of ESWL and stone density ( $p<0.05)$ (Table-IV). 
The stone density and complete clearance of stone showed $78.8 \%$ of the patients with stone density d" 750 HU exhibited complete clearance of stone as opposed to $37.5 \%$ of those with stone density $>750 \mathrm{HU}$. The chance of having complete stone clearance is 6-fold $(95 \% \mathrm{Cl}=1.9-19.4)$ higher in patients with low density stone (d" $750 \mathrm{HU}$ ) than that of patients with high density stone (d" $750 \mathrm{HU})(p=0.002)$. (Table-V).

\section{Table I}

Distribution of patients by their age $(n=96)$

\begin{tabular}{lcc}
\hline Age (Years) $^{*}$ & Number of patients & Percentage \\
\hline$<40$ & 18 & 18.8 \\
$40-50$ & 38 & 39.6 \\
$50-60$ & 31 & 32.3 \\
$\geq 60$ & 9 & 9.4 \\
\hline
\end{tabular}

${ }^{*}$ Mean age $=(49.4 \pm 8.2)$ years; range $=(35-68)$ years ${ }^{\#}$

\section{Table II}

Distribution of patients in relation to number of ESWL session and stone size $(n=96)$

\begin{tabular}{llcc}
\hline $\begin{array}{l}\text { Number of } \\
\text { ESWL session }\end{array}$ & $\begin{array}{l}\text { Stone size } \\
\text { in } \mathrm{cm}\end{array}$ & $\begin{array}{c}\text { Number } \\
\text { of patients }\end{array}$ & Percentage \\
\hline 2 & $0.98-1.20$ & 24 & 25.0 \\
3 & $1.21-1.96$ & 50 & 52.1 \\
$>3$ & $1.97-2.0$ & 22 & 22.9 \\
Stone free rate & & 80 & 83.3 \\
\hline
\end{tabular}

Table II shows that $25 \%$ of the patients underwent 2 sessions where stone size was $0.98 \mathrm{~cm}$ to $1.10 \mathrm{~cm}$. $52.1 \%$ of patients needed three sessions where stone size $1.11 \mathrm{~cm}$ to $1.94 \mathrm{~cm}$ and $22.9 \%$ of patients needed more than 3 sessions where stone size were $1.95 \mathrm{~cm}$ to $2.0 \mathrm{~cm}$. $83.3 \%$ of the patients successfully cleared off stone by ESWL.

Table-III

Association between number of ESWL session and stone density $(n=96)$

\begin{tabular}{llcc}
\hline Number of ESWL sessions & \multicolumn{2}{c}{ Stone density $(\mathrm{HU})$} & p-value \\
& $\leq 750(\mathrm{n}=69)$ & $>750(\mathrm{n}=27)$ & \\
\hline$\leq 3$ & $59(85.5)$ & $15(55.5)$ & $<0.001$ \\
$>3$ & $10(14.5)$ & $12(44.5)$ & \\
Mean \pm SD & $2.7 \pm 0.7$ & $3.2 \pm 1.1$ & \\
\hline
\end{tabular}

\# Data were analyses using Chi-square $\left(\div^{2}\right)$ Test. Figures in the parentheses denote corresponding percentage. Table III shows that $85.5 \%$ of the patients with stone density d" $750 \mathrm{HU}$ needed 3 or less session to have their stone cleared and the rest $(14.5 \%)$ needed $>3$ session with same density. In patients with stone density $>750 \mathrm{HU}, 55.5 \%$ needed 3 or less session and $44.5 \%$ needed $>3$ sessions. $p$-value is significant $(p<0.001)$.

Table-IV

Association between amount of shock wave and stone density $(n=96)$

\begin{tabular}{llcc}
\hline Shock waveף & \multicolumn{2}{c}{ Stone density $(\mathrm{HU})$} & p-value \\
& $\leq 750(\mathrm{n}=69)$ & $>750(\mathrm{n}=27)$ & \\
\hline$\leq 7500$ & $55(79.7)$ & $20(74.1)$ & 0.05 \\
$>7500$ & $14(20.3)$ & $7(25.9)$ & $<0.001$ \\
\hline Average Shock wave & $6689.2 \pm 268.4$ & $9945.2 \pm 375.7$ & $<$ \\
\hline
\end{tabular}

II Data were analyses using Chi-square $\left(\chi^{2}\right)$ Test and were presented as $\mathrm{n}(\%$.) \#Data were analyses using Unpaired t-Test and were presented as mean \pm SD. 
Stone Density Detected By Non Contrast Computed Tomography (Low Dose)

Table -V

Association between stone density and complete clearance of stone ( $n=96)$

\begin{tabular}{|c|c|c|c|c|}
\hline \multirow[t]{2}{*}{ Stone density (HU) } & \multicolumn{2}{|c|}{ Complete clearance } & \multirow{2}{*}{$\begin{array}{c}\text { Risk Ratio } \\
(95 \% \mathrm{Cl} \text { of RR) }\end{array}$} & \multirow[t]{2}{*}{$p$-value } \\
\hline & Yes $(n=80)$ & $\mathrm{No}(\mathrm{n}=16)$ & & \\
\hline$\leq 750$ & $63(78.8)$ & $6(37.5)$ & $6.1(1.9-19.4)$ & 0.002 \\
\hline$>750$ & $17(21.2)$ & $10(62.5)$ & & \\
\hline
\end{tabular}

\# Data were analyses using Chi-square $\left(\chi^{2}\right)$ Test. Figures in the parentheses denote corresponding percentage

\section{Discussion:}

Extracorporeal shockwave lithotripsy is one of the preferred treatment modality for calculus in the upper urinary tract since its introduction[5]. It can clear up to $90 \%$ of stone in adults[15]. However, shockwave lithotripsy monotherapy is not successful in $9.4 \%$ to $26.3 \%$ of renal and proximal ureteral stones[16]. The outcome of ESWL depends on many factors, including stone size, location, composition and fragility, number of shockwave generator, and presence of distal obstruction as well as characteristics of the patient. Failure of ESWL leads to increased costs with requirement of auxiliary procedure to clear the stones. It would be useful if we can predict the stones that will be fragmented by ESWL.

In addition to history, physical examination and urinalysis, routine assessment of a patient presenting with renal colic currently includes NCCT in many centers, though it is not a regular practice in our country. This radiographic modality allows rapid and accurate determination of renal stone. Many investigators have studied the usefulness of NCCT for evaluating urinary calculi and observed that it is superior to traditional imaging such as excretory urography[17]. This study provides compelling data suggesting the importance of measuring $\mathrm{HU}$ in all patients who undergo NCCT to evaluate urinary calculi. By evaluating patients undergoing ESWL for renal calculi determined whether the success of this procedure could be predicted by pretreatment $\mathrm{HU}$ values on NCCT. The ability to assess renal stone characteristics and determine susceptibility to fragmentation is not a new phenomenon. In a study it was compared stone radiodensity with that of spine and concluded that stones are less likely to break if their radiodensity is greater than that of spine[18]. Others studied the opacity of calculi of similar sizes and concluded that fragmentation is less likely with higher opacity[19]. Although these studies provide insight into information needed for therapeutic considerations, they were based on qualitative observations, making them highly subjective and difficult to standardize. CT has long been used clinically to evaluate radiolucent filling defects, using measurements of substance density in $\mathrm{HU}$ to distinguish calculi from tumors or blood clots. The ability of CT scan to detect density differences as low as $0.5 \%$ has been exploited to determine the composition and fragility of urinary stones[13]. The density of stone affects the fragility, which ultimately governs the clinical outcome of ESWL. Therefore, stone opacity were assessed by using a quantitative measurement to evaluate treatment outcomes. $\mathrm{HU}$ calculated on pretreatment NCCT in patients who underwent ESWL provide a simple, easily reproducible and readily available measure of stone opacity. Using this objective study design this study observed that $\mathrm{HU}$ determination on NCCT provides crucial information for the treatment (ESWL) outcome was analyzed.

In the present study the mean age of the patients was 49.4 years with a male preponderance (male to female ratio roughly $3: 1$ ). There were $55.2 \%$ of the patients had left kidney and $44.8 \%$ had right kidney involvement. The mean size of the stone was $1.8 \pm 0.3 \mathrm{~cm}$. There were $62.5 \%$ of the patients had stone in the renal pelvis and $37.5 \%$ in calyceal stone except lower calyx. The mean stone density was $663.7 \pm 69.8 \mathrm{HU}$. $25 \%$ of the patients underwent $2 \mathrm{ESWL}$ sessions, $52.1 \%$ patients underwent 3 sessions and $22.9 \%$ more than 3 sessions. Successful stone clearance by ESWL was found to $80 \%$.

$\mathrm{BMI}$ is an objective measure of obesity, defined as a person's weight in kilograms divided by their height in square meters $\left(\mathrm{kg} / \mathrm{m}^{2}\right)$. The American Society of Clinical Nutrition has defined a BMI of less than 25 as normal, $25-29$ as overweight and 30 or greater as obese ${ }^{[20]}$. In present study BMI has no significant effect to stone clearance by ESWL because in our context patients were no so healthy and bulky like American or European people. Therefore, statistically no significance was found 
in overweight and normal BMI. In a study same result was observed shoewd a significant negative impact of higher BMI on stone free rate after ESWL[21].

Skin to stone distance (SSD) is an easily measured parameter on NCCT which correlates with ESWL efficacy in the treatment of stones in all locations independent of other factors[22]. This study assessed the SSD in all patients, because the SSD calculated on pretreatment NCCT provides a simple, easily reproducible and readily available quantitative measurement. It is the average of three distances (true posterior, $45^{\circ}$ lateral and true lateral) on NCCT. In this current analysis this study included that the mean SSD was $84.2 \pm 2.9$ where d" 3 ESWL sessions is needed and $87.9 \pm 1.8$ where $>3$ session ESWL session is needed to fragment the stone and SSD ranges from $80-88 \mathrm{~mm}$. So it affects the treatment outcome by ESWL. It was described the SSD to be significantly associated with complete stone clearance[21].

In this study, it also assessed that the mean number of shock waves $6689.2 \pm 268.4$ required for stone fragmentation of d" $750 \mathrm{HU}$ and $9945 \pm 375.7$ required > $750 \mathrm{HU}$ stone density respectively. It was concluded that high density renal stone needed greater number of shock waves for complete clearance of stone ${ }^{[23]}$.

The study demonstrated that majority $(78.8 \%)$ of the patients with stone density d" $750 \mathrm{HU}$ experienced complete clearance of stone compared to $37.5 \%$ of those with stone density d" $750 \mathrm{HU}(<0.001) .85 .5 \%$ of the patients with stone density d" $750 \mathrm{HU}$ needed 3 or less session to become stone-free; whereas only $55.5 \%$ of patients with stone density $>750 \mathrm{HU}$ not stone-free in 3 or $<3$ sessions and the rest required 4 or more sessions. In a study it was demonstrated a linear relationship between the calculus density and number of ESWL sessions required. $41(80 \%)$ of patients with stone density of $<$ or $=750 \mathrm{HU}$, needed three or fewer ESWL sessions and $45(88 \%)$ had complete clearance (24). Of patients $41(72 \%)$ with stone density of $>750 \mathrm{HU}$, required more than three ESWL sessions, and 37 (65\%) had complete clearance. The best outcome was in patients with calculus diameters of $<1.1 \mathrm{~cm}$ and mean densities of $<$ or $=750 \mathrm{HU}$; 34 (83\%) needed three or fewer ESWL sessions, and the clearance rate was $90 \%$. The worst outcome was in patients with calculus densities of $>750 \mathrm{HU}$ and diameters of $>1.1 \mathrm{~cm} ; 23$ $(77 \%)$ needed more than 3 ESWL sessions and the clearance rate was only $60 \%$.
Since 1980, NCCT scan has been studied as a possible useful tool to predict stone composition through density measurements (Hounsfield Units). It was stated that NCCT scan could only differentiate the uric acid stones from the rest[25,26]. On the other hand, in a study it was reassures that this instrument can identify uric acid and calcium oxalate stones[27]. Although the development in the technology of endourologic procedures and ESWL increases the management options of renal stones, it also increases the need for more evaluation of their efficacy and indications. Patient, stone and radiographic parameters have been studied as potential predictors of ESWL success. In particular $\mathrm{HU}$ attenuation value of NCCT has been shown to be a potentially useful independent predictor of ESWL outcome. Although CT is associated with greater radiation exposure and costs than plain radiography, NCCT stone characteristics predict ESWL success for renal stone. Patient characteristics are not so much predictive. By knowing the composition of a urinary calculus through density measurement is frequently a key factor in determining its most appropriate management. Whether the stone be amenable to extracorporeal shock wave lithotripsy, or should ureteroscopy or percutaneous nephrolithotomy be attempted can anticipated beforehand. Urine $\mathrm{pH}$, urinary crystals, prior stone history, the presence of urea-splitting organisms, and plain radiography are tools currently used to infer the stone composition[28]. Several in vitro studies have suggested that NCCT can extend these tools by demonstrating measured differences in radio density among different urinary stones[13,29].

The density of stone depends upon its composition and affects the fragility of a calculus, which ultimately governs the clinical outcome of ESWL. Therefore, assessing the density of a calculus before ESWL session starts is of immense significance to increase the efficacy of the procedure and reduce the number of hospital visits and treatment cost.

NCCT has emerged as the modality of choice in the evaluation of acute flank pain. It is safe, rapid, and accurate, with one series reporting $96 \%$ sensitivity, $99 \%$ specificity, and $98 \%$ accuracy in the diagnosis of urolithiasis. Once urinary stone disease is identified, $\mathrm{NCCT}$ is also useful in providing information necessary for the management of the stone.

\section{Conclusion:}

Based on this prospective study we concluded that Low density (d" $750 \mathrm{HU}$ ) renal stone fragmented successfully 
by extra corporeal shock wave lithotripsy but stone of high density (e" $750 \mathrm{HU}$ ) suggest a poor chance of clearance and could help to plane alternative treatment options, thus reduce the burden of the patients..

\section{Refarences:}

1. Curhan GC, 2007. 'Epidemiology of stone disease', Urol Clin North Am, vol. 34, pp. 287-93.

2. Trinchieri A, Coppi F, Montanari E, Del Nero A, Zanetti G, Pisani E, 2000. 'Increase in the prevalence of symptomatic uppere urinary tract stones during the last ten years', Eur Urol 2000, vol. 37, pp. 23-5.

3. Romero V, Akpinar H, Assimos DG, 2010. 'Kidney stones: a global picture of prevalence, incidence and risk factors', Rev Urol, vol. 12, pp. 86-96.

4. Taylor EN, Stampfer MU, Curhan GC, 2005. 'Obesity, weight gain and the risk of kidney stones', JAMA, vol. 293, pp. 455-62.

5. Chaussy C, Brendel W, Schniedt E, 1980. 'Extracorporeally induced destruction of kidney stones by shock waves', Lancet, vol. 2, pp. 12658.

6. Marcinena PG, Peres NB, Liyo J, Ocantos J, Gonzalez M, Jurado A, daels F, 2009. 'CT scan as a predictor of composition and fragility of urinary lithiasis treated with extracorporeal shock wave lithotripsy in vitro', Arch Esp Urol, vol. 62, no. 3, pp. 215-21.

7. Martin TV, Sosa RE, 1998. 'Shock-wave Lithoptripsy', In: Wash PC, Retik AB, Vaughan ED Jr. Wein AJ eds. Campbell's Urology, Philadelphia: WB Saunders Inc., pp. 2735-52.

8. Bon D, Dore B, Irani J, Marroncle M, Albert J, 1996. 'Radographic prognostic criteria for extra-corporeal shock wave lithotripsy: a study of 485 patients', Urology, vol. 48, pp. 556-61.

9. Dretler SP, 1988. 'Stone fragility - a new therapeutic distinction', J Urol, vol. 139, pp. 1124-7.

10. Federie MP, McAninch JW, Kaiser JA, Goodman PC, Roberts J, Mall JC,1981. 'Computed tomography of urinary calculi', AJR Am J Roentgenol, vol. 136, pp. 255-8.

11. Parienty RA, Ducellier R, Pradel J, Lubarano JM, Coquille F, Richard F, 1982. 'Diagnostic value of CT numbers in pelvocalyceal filling defects', Radiology, vol. 145, pp. 743-7.
12. Fielding JR, Steele G, Fox A, Heller H, Loughlin KR, 1997. 'Spiral computerized tomography in the evaluation of acute flank pain: a replacement for excretory urography', J Urol 157, pp. 2071-3.

13. Mostafavi MR, Ernst RD, Saltzman B, 1998. 'Accurate determination of chemical composition of urinary calculi by spiral computerized tomography', J Urol, vol. 159, pp. 673-5.

14. Aruntas, HY. Tekin, I. and Birgul, R. (2010) 'Determining Hounsfield Unit Values of Mortar Constituent by Computerized Tomography', J. Measurement, 43(2), pp. 410-414.

15. Miller, NL. and Lingeman, JE. (2007) 'Management of Kidney Stones', BMJ, 334(3), pp. $468-472$.

16. Ehreth, JT. Drach, GW. and Arnett, ML. (1994) 'Extracorporeal Shockwave Lithotripsy: Multicenter Study of Kidney and Upper Ureter versus Middle and Lower Ureter Treatments', J Urol, 152(6), pp. 1379.

17. Smith, RC. Verga, M. and Dalrymple, N. (1996) 'Acute Ureteral Obstruction: Value of Secondary Signs of Unenhanced Helical CT', AJR Am J Roentgenol, 167(3), pp. 1109-1113

18. Pareek G, Armenakas NA, Fracchia JA, 2003. 'Hounsfield units on computerized tomography predict stone-free rates after extracorporeal shock wave lithotripsy', J Urol, vol. 169, pp. 1679-81.

19. Mattelaer, P. Schroder, T and Fischer, GJ. (1994) 'In Situ Extracorporeal Shockwave Lithotripsy of Distal Ureteral Stones: Parameters for Therapeutic Success', Urol Int. 53(2), pp87 - 91

20. Pareek, G. Armenakas, NA. Panagopoulos, G. Bruno, JJ. and Fracchia, JA. (2004) 'Extracorporeal Shock Wave Lithotripsy Success Based on Body Mass Index and Hounsfield Units', Urology, 65(5), pp. 33-36.

21. Pareek G, Armenakas N et al., 2005. 'Extracorporeal shock wave lithotripsy success based on body mass index and hounsfield units', Urology, vol. 65, pp. 33-36.

22. Patel, T. Kozakowski, K. Hruby, G. Gupta, M. (2009) 'Skin to Stone Distance is an Independent Predictor of Stone-Free Status Following Shockwave Lithotripsy', Journal of Endourology, 23(9), pp. 1383-1385 
23. Joseph P, Mandal A et al., 2002. 'Computed tomography attenuation value of renal calculus: can it predict successful fragmentation of the calculus by extra-corporeal shock wave lithotripsy? A preliminary study', J Urol, vol. 167, pp. 1968-71.

24. Gupta NP, Ansari MS, Kesarvani P, Kapoor A, Mukhopadhyay S, 2005. 'Role of computed tomography with no contrast medium enhancement in predicting the outcome of extracorporeal shock wave lithotripsy for urinary calculi', BJU Int., vol. 95, no. 9, pp. 1285-8.

25. Newhouse J, Prien E et al., 1984. 'Computed tomographic analysis of urinary calculi', Am J Roentgenol, vol. 142, pp. 545-52.
26. Hillman BJ, Drach GW, Tracey P, Gaines JA, 1984. 'Computed tomographic analysis of renal calculi', AJR Am J Roentgenol, vol. 142, no. 3, pp. 549-52.

27. Nakada S, Hoff D et al., 2000. 'Determination of stone composition by noncontrast spiral computed tomography in the clinical setting', Urology, vol. 55, pp. 816-19.

28. Ramakumar S, Patterson DE, LeRoy AJ et al., 1999. 'Prediction of stone composition from plain radiographs: a prospective study', J Endourol, vol. 13, pp. 397-401.

29. Mitcheson HD, Zamenhof RG, Bankoff MS et al., 1983. 'Determination of the chemical composition of urinary calculi by computerized tomography', J Urol, vol. 130, pp. 814-19. 